\title{
Molecular fingerprinting of fusidic acid- and rifampicin-resistant strains of methicillin-resistant Staphylococcus aureus (MRSA) from Malaysian hospitals
}

\author{
A. NORAZAH, V. K. E. LIM, Y. T. KOH, M. Y. ROHANI, H. ZURIDAH*, K. SPENCER†, P. P. NG \\ and A. G. M. KAMEL§
}

Bacteriology Unit, Infectious Diseases Research Centre, Institute for Medical Research, Jalan Pahang, 50588 Kuala Lumpur, *Hospital Umum Sarawak, Jalan Tun Abang Haji Openg, 93590 Kuching, Sarawak, †Hospital Queen Elizabeth, Jalan Mat Salleh, 88590 Kota Kinabalu, Sabah, †Hospital Alor Setar, Jalan Sultan Badlishah, 05590 Alor Setar, Kedah and §Faculty of Allied Health Sciences, National University of Malaysia, Jalan Raja Muda Abdul Aziz, 50300 Kuala Lumpur, Malaysia

\begin{abstract}
The emergence and spread of multiresistant methicillin-resistant Staphylococcus aureus (MRSA) strains, especially those resistant to fusidic acid and rifampicin, in Malaysian hospitals is of concern. In this study DNA fingerprinting by PFGE was performed on fusidic acid- and rifampicin-resistant isolates from Malaysian hospitals to determine the genetic relatedness of these isolates and their relationship with the endemic MRSA strains. In all, 32 of 640 MRSA isolates from 9 Malaysian hospitals were resistant to fusidic acid and rifampicin. Seven PFGE types (A, ZC, ZI, ZJ, ZK, ZL and ZM) were observed. The commonest type was type $\mathrm{ZC}$, seen in $72 \%$ of isolates followed by type $A$, seen in $13 \%$. Each of the other types (ZI, ZJ, ZK, ZL and ZM) was observed in a single isolate. Each type, even the commonest, was found in only one hospital. This suggests that the resistant strains had arisen from individual MRSA strains in each hospital and not as a result of the transmission of a common clone.
\end{abstract}

\section{Introduction}

Fusidic acid has a high degree of activity against Staphylococcus aureus, including methicillin-resistant S. aureus (MRSA). Resistance to fusidic acid can be produced readily by growing $S$. aureus with increasing concentrations of this antibiotic [1]. The development of resistance during treatment with fusidic acid when this antibiotic is used alone is being reported increasingly [2,3]. Rifampicin is also a potent antistaphylococcal agent but its use alone has resulted in rapid emergence of rifampicin-resistant staphylococci [4]. In Malaysia, the treatment of choice for serious

Received 8 March 2002; revised version received 12 Aug. 2002; accepted 15 Aug. 2002.

Corresponding author: Dr A. Norazah (e-mail: norazah@, imr.gov.my).
MRSA infection is vancomycin. However, a combination of fusidic acid and rifampicin is used as an alternative oral regimen for the treatment of bacteraemia and musculoskeletal and cardiovascular infections caused by MRSA. Fusidic acid and rifampicin are used in combination to prevent the emergence of resistance which may occur if these antibiotics are used individually. In Malaysian hospitals, the resistance rates for fusidic acid and rifampicin individually were reported to be within the range $3-5 \%$ in the years 1992-1996 [5]. As these antibiotics provide an alternative or switch therapy to vancomycin in Malaysia, it is important to know the resistance rates of MRSA to both these antibiotics. The main objectives of this study were to determine the resistance rates to fusidic acid and rifampicin among MRSA isolates in Malaysian hospitals and to determine the genetic relatedness of these isolates and their relationship with the endemic strains in the hospitals by pulsed-field gel electrophoresis (PFGE). 


\section{Materials and methods}

\section{Bacterial strains}

The MRSA isolates were obtained from nine government hospitals in Malaysia. The hospitals were Alor Star (HAS), Taiping (HTA), Kuala Lumpur (HKL), Universiti Kebangsaan Malaysia (HUKM), Seremban (HSE), Sultanah Aminah (HSA), Kota Bharu (HKB), Sarawak (HUS) and Queen Elizabeth (HQE). All these hospitals except HKL and HUKM are situated in different states in Malaysia.

The isolates were from clinical samples and were identified in the microbiology laboratory of each of the hospitals. They were collected from 1997 until 1999. Randomly selected isolates from these laboratories were sent to the Bacteriology Unit, Institute for Medical Research, Kuala Lumpur.

\section{Antibiotic susceptibility testing}

The MRSA isolates were subjected to antibiotic disk susceptibility testing following the guidelines of the National Committee for Clinical Laboratory Standards (NCCLS) [6]. MRSA isolates that were resistant to fusidic acid and rifampicin by the disk susceptibility test were then tested for fusidic acid and rifampicin MIC following the NCCLS recommendations. Serial two-fold dilutions of fusidic acid (Leo Pharmaceutical Products, Denmark) were added to molten MuellerHinton II agar (BBL, USA) to make up concentrations ranging from $256 \mathrm{mg} / \mathrm{L}$ to $1 \mathrm{mg} / \mathrm{L}$. Serial dilutions were also made for rifampicin (Sigma Aldrich) to give concentrations ranging from $256 \mathrm{mg} / \mathrm{L}$ to $1 \mathrm{mg} / \mathrm{L}$. Isolates were considered to be resistant to fusidic acid if the MIC was $>2 \mathrm{mg} / \mathrm{L}$ [7] and resistant to rifampicin if the $\mathrm{MIC}$ was $\geqslant 4 \mathrm{mg} / \mathrm{L}$ [8].

\section{PFGE}

For each isolate tested, an isolated colony was inoculated into $5 \mathrm{ml}$ of tryptic soya broth and incubated overnight at $37^{\circ} \mathrm{C}$. The broth culture was then adjusted to a concentration of $1 \times 10^{9} \mathrm{cfu} / \mathrm{ml}$. After washing in TE1 buffer (10 mM Tris-HCl, $50 \mathrm{~mm}$ EDTA, $\mathrm{pH} 7.5$ ), $200 \mu \mathrm{l}$ of the bacterial suspension were added to an equal volume of low-melting point agarose $2 \% ; 6 \mu \mathrm{l}$ of lysostaphin $(1 \mathrm{mg} / \mathrm{ml})$ were added immediately and the suspension was mixed well before being allowed to solidify in a plug mould. The gel plugs were then incubated overnight at $55^{\circ} \mathrm{C}$ in $2 \mathrm{ml}$ of $\mathrm{ES}$ buffer (N-laurylsarcosine $1 \%$ in $0.5 \mathrm{ml}$ of EDTA, $\mathrm{pH} 8.0$ ) containing proteinase $\mathrm{K}(10 \mathrm{mg} / \mathrm{ml})$ with gentle shaking. The plugs were washed three times with TE2 (10 mM Tris, $1 \mathrm{mM}$ EDTA, pH 8.0) for $20 \mathrm{~min}$ each on ice. A slice of the plug $(2.5 \mathrm{~mm})$ was cut and digested with 40 units of $S m a \mathrm{I}$ and incubated at $30^{\circ} \mathrm{C}$ overnight. The DNA samples were then electrophoresed in agarose $1.2 \%$ with a contour-clamped homogeneous electric field (CHEF-DRIII, BioRad). The pulse times used were $5-15 \mathrm{~s}$ for $8 \mathrm{~h}$ followed by $15-25 \mathrm{~s}$ for $10 \mathrm{~h}$. The gel was then stained with ethidium bromide and photographed under UV light with Gel Doc 1000 (BioRad). DNA fragment patterns that differed by three or less bands were interpreted as sharing a common PFGE type (designated by capital letters); subtypes were defined as variants with one-to-three different DNA fragments $[9,10]$.

\section{Results}

A total of 640 MRSA isolates was investigated by antibiotic disk susceptibility testing, of which 32 isolates were found to be resistant to fusidic acid and rifampicin. The resistance was confirmed by determination of the MIC of fusidic acid and rifampicin; all 32 isolates were confirmed to be resistant to both antibiotics with $\mathrm{MICs} \geqslant 4 \mathrm{mg} / \mathrm{L}$.

The hospitals with isolates resistant to both these antibiotics were HQE, HUKM, HAS and HTA; no such resistant isolates were observed in the other hospitals. Twenty-three of $151 \mathrm{HQE}$ isolates tested, 5 of 62 HUKM isolates, 3 of 42 HAS isolates and 1 of 51 HTA isolates were resistant to fusidic acid and rifampicin. They were isolated from skin and wound swabs (47\%), pus $(28 \%)$, blood $(9 \%)$, tracheal aspirates $(6 \%)$, sputum $(3 \%)$, urine $(3 \%)$ and tissue $(3 \%)$. The 23 isolates from HQE were from the medical ward (10), surgery (4), orthopaedic (4), intensive care unit (1), burns unit (1) and unknown (3). All the isolates from HUKM were from the surgical ward.

\section{PFGE of fusidic acid- and rifampicin-resistant MRSA strains}

Seven major PFGE types were found among the 32 isolates that were subjected to PFGE - types A, ZC, ZI, ZJ, ZK, ZL and ZM (Fig. 1). Type ZC was found in 23 isolates and type $\mathrm{A}$ in 4 isolates. The other five types occurred only once.

Type ZC was observed in isolates from HQE. On prior screening of 40 MRSA isolates randomly selected from this hospital, 10 of the isolates were of type $\mathrm{ZC}$, of which 9 were resistant to both fusidic acid and rifampicin, while one was resistant to fusidic acid only. All the isolates with type ZC were also resistant to five other antibiotics - erythromycin, gentamicin, tetracycline, sulfamethoxazole-trimethoprim and ciprofloxacin.

Resistant isolates of types A and ZI were from HUKM. Type A, seen in four of the five MRSA isolates resistant to fusidic acid and rifampicin, is also the predominant MRSA type seen in Malaysian hospitals [11]. Other type A isolates from HUKM were sensitive to fusidic acid and rifampicin. The fifth isolate (type 


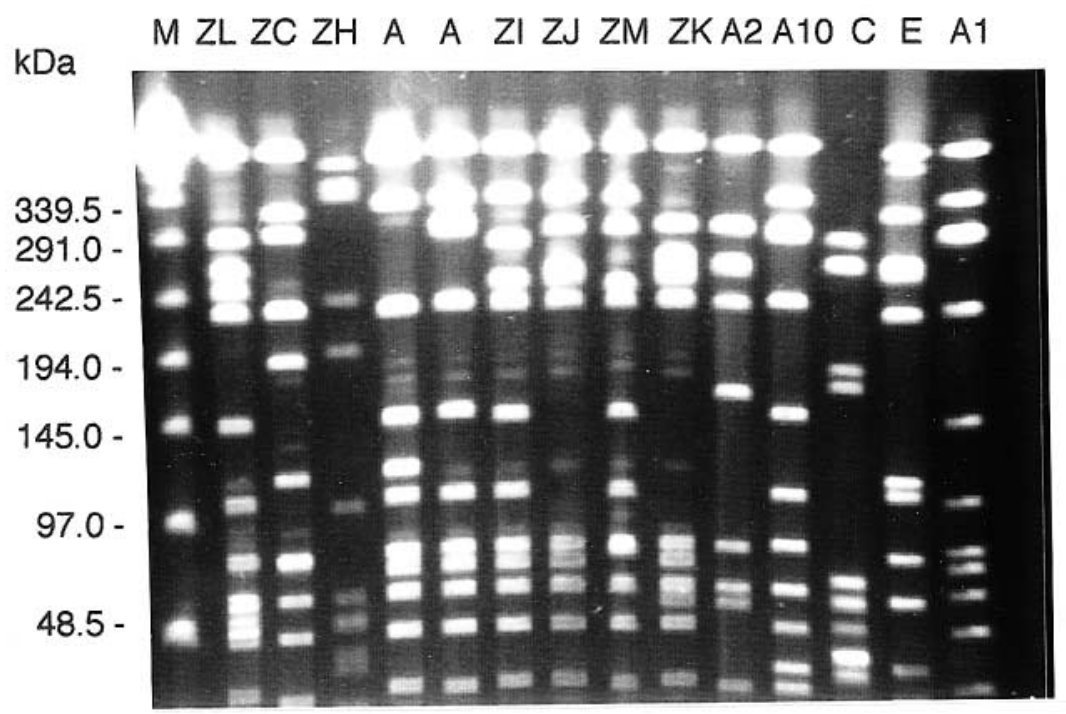

Fig. 1. PFGE of fusidic acid- and rifampicin-resistant MRSA. Two isolates of PFGE type A are shown, which differ by two bands. $\mathbf{M}$, PFGE marker; A2, A10, $\mathbf{C}, \mathbf{E}$ and A1, representatives of common PFGE profiles of MRSA strains that are sensitive to fusidic acid and rifampicin.

ZI) had a PFGE pattern that differed from type A by $>10$ bands. This pattern was not observed on prior screening of endemic MRSA strains in HUKM.

Three PFGE types (ZJ, ZK and ZL) were observed for the three isolates that were resistant to fusidic acid and rifampicin from HAS. These PFGE types were not seen in 28 other randomly selected isolates of MRSA from HAS that were sensitive to fusidic acid and rifampicin. Furthermore, these three PFGE types were not closely related to one another, showing that they had arisen from individual clones. The only isolate from HTA was of PFGE type ZM, which was not observed among the resistant isolates from other hospitals.

The PFGE pattern of the isolates that were resistant to fusidic acid and rifampicin showed that the majority of such strains arose from separate and unique clones and were not due to an increase in inter-hospital transmission.

\section{Discussion}

Besides having the advantage of oral administration, fusidic acid and rifampicin penetrate tissues better than glycopeptides. The appearance of resistance to either of these antibiotics will adversely affect effective oral treatment of MRSA infections. Rifampicin and fusidic acid should be used in combination when treating MRSA infections to prevent the emergence of resistance to either of these antibiotics.

Most of the fusidic acid- and rifampicin-resistant isolates in this study were of type $\mathrm{ZC}$, isolated from HQE, which is located in East Malaysia. As this was the only PFGE type that exhibited fusidic acid and rifampicin resistance in this hospital, it is possible that these isolates represent part of a larger outbreak with this strain in the hospital. These isolates were from the medical, surgical, orthopaedic, burns and ICU wards during the period Nov. 1997 to July 1999. Thus, strains with this type have been present in the wards since 1997. An earlier analysis of 40 isolates from HQE showed that the strains with other typing patterns did not exhibit resistance to fusidic acid and rifampicin. One isolate (PFGE type ZC) was resistant to fusidic acid but sensitive to rifampicin. This clone, initially sensitive to both antibiotics, may have undergone mutation and acquired resistance to fusidic acid. No information was available on the usage of fusidic acid and rifampicin in this hospital but the emergence of such resistance has been associated with monotherapy [12].

Strains in HUKM and HAS were heterogeneous in that more than one type was observed among their isolates. The HUKM isolate with type A had three subtypes, showing that these isolates were closely related and probably originated from the same clone. The three different types observed in HAS showed that the resistant strains originated from three different clones.

However, resistance to both these antibiotics was uncommon in other hospitals. In a study by Schmitz et al. [13], it was shown that the development of higher incidence rates of MRSA was not the result of hypermutability of target genes or a faster emergence of different mutations but rather the consequence of clonal spread of multiresistant MRSA.

The information gained from the typing of fusidic acidand rifampicin-resistant isolates can help the hospital infection control teams understand the epidemiology of 
this organism in their institutions. The spread of these strains can be tracked and further transmission prevented by isolating the affected patients and applying appropriate barrier nursing techniques. Surveillance of these strains should be carried out to prevent further spread of the clone. Increased resistance to rifampicin and fusidic will further reduce the already limited treatment options for MRSA infections.

The results of the PFGE fingerprinting showed that the fusidic acid- and rifampicin-resistant strains appear to arise from individual MRSA clones in each hospital. The unique fingerprints of these strains suggest that the strains may have arisen from antibiotic selection pressure. As the emergence of resistance has been associated with monotherapy, the education of doctors in Malaysia on the appropriate prescribing of rifampicin and fusidic acid is crucial to prevent further emergence of these resistant strains.

This work was supported by the Ministry of Science and Technology, Malaysia (IRPA Grant number 06-05-01-0127).

\section{References}

1. O’Brien FG, Botterill CI, Endersby TG, Lim RLG, Grubb WB, Gustafson JE. Heterogeneous expression of fusidic acid resistance in Staphylococcus aureus with plasmid or chromosomally encoded fusidic acid resistance genes. Pathology 1998; 30: 299-303.

2. Ravenscroft JC, Layton A, Barnham M. Observations on high levels of fusidic acid resistant Staphylococcus aureus in
Harrogate, North Yorkshire, UK. Clin Exp Dermatol 2000; 25: $327-330$

3. Chang SC, Hsieh SM, Chen ML, Sheng WH, Chen YC. Oral fusidic acid fails to eradicate methicillin-resistant Staphylococcus aureus colonization and results in emergence of fusidic acid-resistant strains. Diagn Microbiol Infect Dis 2000; 36: $131-136$.

4. Kucers A, Bennet MK. Rifampicin (Rifampin). In: The use of antibiotics, 4th edn. London, William Heinmann Medical Books. 1987: 914-970.

5. Rohani MY, Zainuldin MT, Koay AS, Lau MG. Antibiotic resistance patterns of bacteria isolated in Malaysian hospitals. Int Med J 1999: 47-51.

6. National Committee for Clinical Laboratory Standards. Performance standards for antimicrobial disk susceptibility tests. PA, NCCLS. 1998.

7. Toma E, Barriault D. Antimicrobial activity of fusidic acid and disk diffusion susceptibility testing criteria for gram-positive cocci. J Clin Microbiol 1995; 33: 1712-1715.

8. Thornsberry C, McDougal LK. Successful use of broth microdilution in susceptibility tests for methicillin-resistant (heteroresistant) staphylococci. J Clin Microbiol 1983; 18: 1084-1091.

9. Tenover FC, Arbeit RD, Goering RV et al. Interpreting chromosomal DNA restriction patterns produced by pulsedfield gel electrophoresis: criteria for bacteria strain typing. J Clin Microbiol 1995; 33: 2233-2239.

10. Goering RV, Tenover FC. Epidemiological interpretation of chromosomal macrorestriction fragment patterns analysed by pulsed-field gel electrophoresis. J Clin Microbiol 1997; 35: $2432-2433$.

11. Norazah A. Studies on the molecular epidemiology of MRSA strains in Malaysia. $\mathrm{PhD}$ thesis, National University of Malaysia, 2002.

12. Whitby M. Fusidic acid in the treatment of methicillin-resistant Staphylococcus aureus. Int J Antimicrob Agents 1999; 12 Suppl 2: S67-S71.

13. Schmitz FJ, Fluit AC, Hafner D et al. Development of resistance to ciprofloxacin, rifampin and mupirocin in methicillin-susceptible and -resistant Staphylococcus aureus isolates. Antimicrob Agents Chemother 2000; 44: 3229-3231. 